\title{
Ansiedad estado precompetitiva en judocas
}

\section{Carlos MONTERO CARRETERO ${ }^{1 *}$, Juan A. MORENO-MURCIA ${ }^{1}$, Inmaculada GONZÁLEZ PONCE $^{2}$, Juan J. PULIDO GONZÁLEZ ${ }^{2} \&$ Eduardo M. CERVELLÓ GIMENO ${ }^{1}$}

\author{
1 Universidad Miguel Hernández de Elche (España) \\ 2 Universidad de Extremadura (España)
}

Recepción: 16/01/2012; Aceptación: 15/05/2012; Publicación: 15/06/2012.

\begin{abstract}
Resumen
Objetivos: Entendiendo la ansiedad desde una perspectiva multidimensional, el propósito de este estudio fue comprobar las propiedades psicométricas del cuestionario Competitive State Anxiety Inventory-2 (CSAI-2) en judokas españoles, y examinar las diferencias de ansiedad estado precompetitiva en función de su nivel deportivo, edad y género.

Método: Participaron en el estudio 128 judokas de nivel amateur y alto rendimiento. Las variables medidas antes de la competición fueron: intensidad y dirección de la ansiedad somática, cognitiva y autoconfianza del CSAI-2.

Resultados: Los resultados obtenidos muestran que el cuestionario administrado presentó unas propiedades psicométricas aceptables, y que existen diferencias en la dirección de la ansiedad somática y cognitiva para la edad, así como en la intensidad de la autoconfianza para el nivel deportivo. Las implicaciones que estos resultados pueden tener en el proceso de entrenamiento y en la competición se discuten en el documento.
\end{abstract}

Palabras clave: Ansiedad precompetitiva, autoconfianza, judo.

\section{Precompetitive state anxiety in judo}

\begin{abstract}
Aim: The objective of this study was to analyze the psychometric properties of the Competitive State Anxiety Inventory-2 (CSAI-2) in Spanish judokas, and calculate differences in pre-competitive state anxiety due the sport level, age and gender. We analyze these relationships using a multidimensional anxiety perspective.

Method: A sample of 128 judokas from amateur to high performance level participated in our study. The intensity and directional somatic anxiety, cognitive anxiety and self confidence of the CSAI-2 were measured.

Results: The results show that the questionnaire administered showed acceptable psychometric properties, and there are differences in directional somatic and cognitive anxiety for age, and in intensity self confidence for sport level. The implications of these findings for the process of training and competition are discussed in the document.
\end{abstract}

Keywords: Pre competitive anxiety, self confidence, judo.

\section{Ansiedade estado pré-competitivo e judocas}

\section{Resumo}

Objectivos: Entendendo a ansiedade numa perspectiva multidimensional, o propósito deste estudo foi comprovar as propriedades psicométricas do questionário Competitive State Anxiety Inventory-2 (CSAI-2) nos judocas espanhóis e examinar as diferenças de ansiedade estado pré-competitiva em função do seu nível desportivo, idade e género.

Método: Participaram neste estudo 128 judocas de nível amador e de alto rendimento. As variáveis medidas antes da competição foram: intensidade e direcção somática, cognitiva e auto-confiança do CSAI-2.

Resultados: Os resultados obtidos mostram que o questionário administrado apresentou umas propriedades aceitáveis e que existem diferenças na direcção da ansiedade somática e cognitiva para a idade, assim como na intensidade da auto-confiança para o nível desportivo. As implicações que estes resultados podem ter no processo de treino e na competição são discutidos no documento.

Palavras-chave: Ansiedade pré-competitiva, autoconfiança, judo.

*E-mail: cmontero@umh.es. Dirección: Centro de Investigación del Deporte de la Universidad Miguel Hernández de Elche. Avenida de la Universidad, s/n. 03202. Elche (España). 


\section{1.- Introducción}

La ansiedad ha sido entendida, tradicionalmente, como un estado emocional negativo (Weinberg \& Gould, 2010). Landers y Boutcher (1986) la definen como una reacción emocional displacentera que acompaña al arousal del sistema nervioso autónomo, y es considerada una condición emocional desadaptativa. Otros autores (Cervantes, Rodas, \& Capdevila, 2009; Gould, 1987; Gould, Greenleaf, \& Krane, 2002; Martens, 1977; Santos-Rosa, 2003; Smith, 2008) consideran la ansiedad como una respuesta psicológica que se produce como consecuencia de la discrepancia entre la demanda del ambiente y la capacidad de respuesta, que siempre irá acompañada de un alto grado de activación psicofisiológica. Pero no todos los investigadores se refieren a ella como un factor con connotaciones negativas. Algunos consideran que puede ser positiva o indiferente hacia la ejecución deportiva, dependiendo de aspectos como la personalidad del deportista, la dificultad de la tarea o el nivel de habilidad del individuo (Brandao, 1995; Cervelló, Santos-Rosa, Jiménez, Nerea, \& García, 2002).

Este constructo se puede entender desde una perspectiva multidimensional (Cheng, Hardy, \& Markland, 2009), diferenciando entre intensidad y dirección de la ansiedad (Jones, 1991; Jones \& Hanton, 2001; Hanton \& Swain, 1994; Jones, Swain, \& Hardy, 1993), ansiedad rasgo y estado (Spielberger \& Díaz, 1975), o entre somática (Davidson \& Schwartz, 1976; Martens, Burton, Rivkin, \& Simo, 1990a) y cognitiva (Eysenck \& Calvo, 1992; Liebert \& Morris, 1967; Spielberger, 1980).

La intensidad de la ansiedad puede entenderse como la magnitud de los síntomas que produce, mientras la dirección hace referencia a la interpretación que hace el sujeto de esos síntomas en cuanto a su rendimiento futuro (Jones, 1991). Hay sujetos que entienden determinados niveles de ansiedad como beneficiosos para su rendimiento en la actividad a la que se enfrentaran, mientras otros interpretan esos niveles de ansiedad como debilitadores del rendimiento. Algunos estudios demuestran que el nivel de habilidad podría mediar la interpretación que los deportistas hacen de los síntomas de la ansiedad que experimentan, de modo que los de mayor nivel considerarían dichos síntomas como más facilitadores del rendimiento futuro (Jones, Hanton \& Swain, 1994; Jones \& Swain, 1995; Jones et al., 1993; Raudsepp \& Kais, 2002).

De otro lado, la ansiedad estado es una condición o estado emocional transitorio del organismo humano, que se caracteriza por sentimientos de tensión y aprensión subjetivos conscientemente percibidos, y por un aumento de la actividad del sistema nervioso autónomo. Los estados de ansiedad pueden variar en intensidad y fluctuar a través del tiempo (Spielberger \& Díaz, 1975). La ansiedad rasgo se refiere a las diferencias individuales, relativamente estables, en la propensión a la ansiedad, es decir, a las diferencias entre las personas en la tendencia a responder a situaciones percibidas como amenazantes con elevaciones en la intensidad de la ansiedad estado (Spielberger \& Díaz, 1975). Cuando el motivo que genera la ansiedad es la competición deportiva, se habla de ansiedad rasgo o estado competitiva (Martens, 1977).

La ansiedad se manifiesta de manera similar a otras emociones, conjugando tres sistemas de respuesta o dimensiones (Borkovek, 1976). Las preocupaciones respecto a la ejecución y falta de habilidad para concentrarse se conocen como ansiedad cognitiva (Eysenck \& Calvo, 1992; Liebert \& Morris, 1967). A esta dimensión pertenecen las experiencias de miedo, pánico, alarma, inquietud, aprensión, obsesiones, y pensamientos intrusivos de tipo catastrófico. La ansiedad somática son las percepciones de los síntomas corporales causados por la activación del sistema nervioso autónomo, tales como aceleración del ritmo cardíaco, sudoración, etc. en situaciones de ansiedad cognitiva (Hardy, Jones, \& Gould, 1996; Cheng, Hardy, \& Markland, 2009; Martens et al., 1990a). Es la dimensión de la ansiedad que provoca cambios y activación fisiológica de tipo externo (sudoración, dilatación pupilar, temblor, tensión muscular, palidez facial, entre otros), interno (aceleración del ritmo cardíaco y respiratorio, descenso de la salivación, entre otros), e involuntarios o parcialmente voluntarios (palpitaciones, temblor, vómitos, desmayos, entre otros). Existe una última dimensión conocida como autoconfianza, relacionada con la creencia de los sujetos de estar preparados para la situación a la que se enfrentan (López Torres, Torregrosa, \& Roca, 2007; Tsopani, Dallas, \& Skordilis, 2011). La autoconfianza sería prácticamente lo opuesto a la ansiedad cognitiva.

La investigación efectuada en el campo de la ansiedad competitiva en el deporte, ha mostrado la existencia de una serie de elementos tanto personales como situacionales relacionados 
con la aparición de la ansiedad previa a las competiciones (Cervelló et al., 2002). Según esta perspectiva (cognitivo-afectiva) los elementos responsables de la aparición de la ansiedad no serán solo personales, sino que también influyen aspectos tales como el tipo de deporte o la complejidad de la tarea (Burton, 1998). La competición de judo transcurre en situaciones que cambian constantemente, donde el deportista está en contacto directo con su contrincante, que se opone de forma directa a las acciones, en una situación de contra comunicación. La incertidumbre en cuanto al combate de judo es muy elevada, teniendo en cuenta que es en gran parte imprevisible lo que el oponente vaya a hacer en cada momento. El sistema de puntuación hace que una técnica pueda terminar el combate por ippon en un instante, sin oportunidad de poder enmendar el error. Por otro lado, los judocas que se preparan para un campeonato, a menudo se juegan el resto de la temporada en dicho evento, de cuyo resultado depende que tengan oportunidad de salir a competir a futuros torneos. Todos estos condicionantes hacen que la competición de judo pueda producir altos niveles de ansiedad en los deportistas que participantes en ella.

\section{1.- Relación entre ansiedad y ejecución}

Existen diferentes teorías que han tratado de explicar la relación entre la ansiedad y el rendimiento. Spence y Spence (1966) determinaron que a mayor nivel de activación fisiológica mayor sería el rendimiento, entendiendo una relación lineal en lo que se llamó la Teoría del Impulso. En cambio la hipótesis de la U invertida (Landers \& Boutcher, 1986) sostiene que el rendimiento aumenta a medida que aumenta la ansiedad hasta un cierto punto, a partir del cual el rendimiento comenzará a descender tanto como continúe aumentando esa activación fisiológica. Por otro lado, la Teoría de la Catástrofe (Hardy \& Facey, 1987) indica que una vez que se alcanza un cierto nivel de ansiedad el rendimiento empieza a decrecer paulatinamente, pero que llegados a un cierto punto se produce un desplome repentino del mismo, una "catástrofe en el rendimiento". Finalmente, otros autores sostienen que existen zonas de óptimo rendimiento, o zonas de funcionamiento óptimo (ZOF) (Hanin, 1980, 1993, 1997). Según esta teoría los deportistas tienen una zona de estado óptimo de la ansiedad en el que se produce el máximo rendimiento, de forma que fuera de dicho rango de ansiedad, el rendimiento es inferior. Algunos estudios realizados con karatekas apoyan dicha teoría (Ruiz \& Hanin, 2004a; 2004b).

Woo (1996) realizó un estudio con judocas de élite coreanos, en el que concluyó que la teoría para explicar la relación entre ansiedad y rendimiento que más se acercaba a los resultados obtenidos en ese estudio era la Teoría de la Catástrofe, tras realizar entrevistas en las que los judocas recordaron sus mejores y peores rendimientos relacionados con la ansiedad que habían experimentado.

La necesidad de contemplar la ansiedad bajo un prisma multidimensional, conduce a la Teoría Multidimensional de la Ansiedad (MAT) (Burton, 1988; Martens et al., 1990) según la cual la ansiedad cognitiva afecta en el rendimiento o en la ejecución de forma diferente a como lo hace la ansiedad somática (Davidson \& Schwartz, 1976; Gould, Petlichkoff, \& Weinberg, 1984; Jones, 1995; Liebert \& Morris, 1967; Martens, et al., 1990). De este modo, ante una misma situación estresora, algunos individuos responden con un aumento de la ansiedad cognitiva, mientras otros lo hacen con un aumento de la ansiedad somática. Es más, el mismo individuo puede responder de forma diferente según cuál sea la situación. La MAT predice que la ansiedad estado cognitiva influye en el rendimiento de forma lineal y negativa, mientras la ansiedad estado somática y el rendimiento se relacionan en forma de U-invertida (Burton 1988; Martens et al., 1990b; Márquez, 2004). Además, según la MAT, la autoconfianza tendría una relación lineal positiva con el rendimiento. No obstante algunos estudios no dan soporte, o lo hacen parcialmente, a lo que expone la teoría. Este es el caso de los trabajos realizados con tiradores de pistola (Gould \& Krane, 1992), futbolistas (Rodrigo et al., 1990), Hockey (Mynard \& Howe, 1987) o atletas de resistencia (Hammermeister \& Burton, 1995). Aunque no abundan, también hay algunas investigaciones al respecto que incluyen aspectos de intervención (Maynard \& Cotton, 1993; Maynard et al., 1995). Los resultados obtenidos indican que el tipo de técnicas de control de la ansiedad que se emplean dependen del tipo de ansiedad estado que el sujeto experimenta. Así, un estudio reciente con metodología experimental (Hatzigeorgiadis et al., 2009) concluye que las autoinstrucciones pueden ser una técnica eficaz para reducir la ansiedad cognitiva y aumentar la autoconfianza. 
Respecto a la autoconfianza, generalmente niveles altos de esta variable conllevan una sensación de poder superar el reto, incluyendo sus sensaciones ansiógenas, convirtiéndose esa autoconfianza en el propio mecanismo de defensa ante los síntomas de la ansiedad, tal y como se concluye en algunas investigaciones (López-Torres et al., 2007). Hanton, Mellieau, y Hall (2004) ya señalaban la autoconfianza como una cualidad esencial en los deportistas de élite, por el efecto que esta tiene sobre la ansiedad estado precompetitiva, tal y como se había señalado en estudios que versan sobre la interpretación que los deportistas hacen de los síntomas de la ansiedad previa a la competición en relación con la autoconfianza (Bandura, 1982; Hanton \& Connaughton, 2002; Jones et al., 1994). A este respecto algunos autores defienden que la dirección de la ansiedad predice mejor el rendimiento que la intensidad de esta (Santos-Rosa, 2003). Igualmente la autoconfianza se ha estudiado como elemento mediador de otros procesos respecto al rendimiento deportivo (Martens et a., 1990), mostrándose siempre una relación positiva entre ambas variables (Craft et al., 2003). Algunos autores apuntan que altos niveles de autoconfianza reportan en los sujetos un aumento de la motivación y el esfuerzo de los deportistas para desarrollar la tarea a la que se enfrentan, aumentando su concentración aún en condiciones de alta ansiedad (Eysenck \& Calvo, 1992; Hardy, 1997). El estudio cualitativo de Hanton et al. (2004) da soporte a los estudios anteriores, en tanto en cuanto, los deportistas que manifiestan alta autoconfianza junto con altos valores de ansiedad competitiva, interpretan los síntomas de la ansiedad como facilitadores de su rendimiento futuro, mientras cuando esos valores de ansiedad se presentan conjuntamente con bajos niveles de autoconfianza, los sujetos consideran que esa ansiedad facilitará un bajo rendimiento futuro.

\section{2.- Investigación sobre ansiedad en judocas}

La ansiedad ha sido estudiada en judocas con el fin de averiguar qué papel juega sobre el rendimiento, cómo varía a lo largo de una competición, cómo afecta a los deportistas que tienen problemas de peso, y cómo varía en función de la importancia del campeonato o en función de la edad y experiencia de los deportistas.

En un estudio antiguo realizado con judocas brasileños (Moraes, 1987) no se encontraron diferencias en la ansiedad medida antes y después de una competición, pero sí diferencias por género, de modo que los chicos obtuvieron mayores valores en la dimensión autoconfianza que las chicas. En cambio, en otro trabajo llevado a cabo con judocas de élite coreanos (Woo, 1996) los resultados mostraron que no existían diferencias significativas entre grupos por género, mientras los marcadores de ansiedad estado (somática y cognitiva) y rasgo eran más elevados en las peores experiencias de rendimiento. Por su parte, Kuuseok y Ratassepp (2001), en una investigación realizada con judocas estonios, desvelaron que un temperamento inestable correlacionaba positiva y significativamente con niveles de ansiedad más elevados. Además, los judocas más jóvenes e inexpertos mostraron niveles de ansiedad más elevados que los de mayor edad igual que sucediera en otro estudio realizado con judocas españoles infantiles y cadetes (Hernández et al., 2008) en el que no se observaron diferencias en la autoconfianza.

Salvador et al. (2003) comprobaron en una muestra de judocas que los niveles ansiedad estado fueron más elevados en la competición, de lo que venían siendo habituales en las sesiones de entrenamiento, al igual que sucediera en otro estudio con judocas alemanes (Marahrens \& Keil, 2004) en el que además se describió el síndrome del "campeón de entrenamiento" caracterizado por buenos rendimientos en entrenos y pobres rendimientos en competición donde las intensidades de la ansiedad aumentaban notablemente.

En el intento de ver cómo afectan los problemas de peso a la ansiedad de los judocas, Nasioudis, Kabitsi, Kabitsis, Harahousou, y Lailoglou (2005) comparan ésta variable en judocas de alto nivel griegos, separados en dos grupos diferentes; uno que tenía problemas y otro que no. Los resultados revelaron que no existían diferencias intergrupos en la ansiedad estado medida antes del pesaje, pero sí tras el mismo. Los que tenían problemas de peso, mostraron un mayor descenso de la ansiedad cognitiva y somática que los que no tenían problemas, cuando esta fue medida tras el pesaje. En cambio, la autoconfianza aumentó, tras dar el peso, en mayor medida en ese grupo con problemas que en el que estaba compuesto por judocas que daban el peso bien. Esto muestra la 
importancia que puede tener para algunos judocas el momento del pesaje, y cómo ese momento podría suponer un antes y un después en su estado psicológico.

Los resultados de otro estudio (Moya-Albiol, Salvador, Costa, Martínez-Sanchis, GonzálezBono, Ricarte, \& Arnedo, 2001) realizado con una muestra de judocas y jugadores de balonmano de alto nivel por un lado, así como por personas que practican actividad física con fines saludables, muestran que los deportistas de alto nivel presentaban mejores valores en los parámetros de carácter fisiológico, y no se encontraron diferencias significativas entre grupos en la ansiedad rasgo medida antes de una prueba en el cicloergómetro, ni en la ansiedad estado medida tras la misma.

En cambio, en otro estudio (Filaire et al., 2001), llevado a cabo con 12 judocas franceses de nivel interregional se encontraron que los judocas mostraron valores de ansiedad somática y cognitiva significativamente mayores cuando abordaban las competiciones de nivel más elevado mientras la autoconfianza era más baja.

\section{3.- Instrumentos más utilizados para medir la ansiedad en el deporte}

Tradicionalmente se han empleado autoinformes para la evaluación de la ansiedad, dentro de los cuales uno de los más empleados en psicología del deporte ha sido la Escala de Ansiedad Estado-Rasgo (STAI) de Spielberger (1989). El STAI está compuesto por dos escalas de 20 ítems cada una, destinadas a medir la ansiedad rasgo y la ansiedad estado.

En el intento de crear una herramienta útil y más específica para el ámbito deportivo, se desarrollaron otras escalas como el SCAT (Sport Competition Anxiety Test), el CSAI-2 (Competitive State Anxiety Inventory) de Martens (1977), Martens, Burton, Rivkin, y Simo (1980) y Martens et al. (1990b), y el SAS (Sport Anxiety Scale) de Smith, Smoll, y Schutz (1990).

El SCAT intenta medir la ansiedad rasgo en la línea de lo teorizado anteriormente por Spielberger (1972), y "su importancia reside en ser el pionero en dotarse de un diseño específico para poblaciones de deportistas" (Jaenes \& Caracuel, 2005). Por su parte, la Escala de Ansiedad Deportiva (SAS) fue creada teniendo en cuenta la multidimensionalidad de la ansiedad para medir las reacciones somáticas, las preocupaciones cognitivas y las dificultades atencionales en contextos deportivos (Santos Rosa, 2003).

Con el fin de medir la ansiedad desde el prisma de las teorías multidimensionales, el CSAI-2 nace tras una revisión (Martens et al., 1982) del CSAI, en el cual ya se evaluaba ansiedad cognitiva y somática generales en situaciones precompetitivas. Tras varias investigaciones se llegó a la conclusión de que la ansiedad estado cognitiva se dividía en dos factores claramente diferenciados. Estos dos factores son la autoconfianza estado (considerada por los ítems positivos) y la ansiedad cognitiva estado (considerada en los ítems negativos). De este modo la última versión del CSAI-2 se compone finalmente de un total de 17 ítems, repartidos en 3 escalas, que son: ansiedad somática estado, ansiedad cognitiva estado y autoconfianza. Teniendo en cuenta la dimensión direccional de la ansiedad, el CSAI-2 está compuesto por otra escala tipo Likert (de $-3 \mathrm{a}+3$ ) en la que el deportista interpreta si la intensidad de la ansiedad que experimenta será facilitadora u obstaculizadora en su rendimiento próximo. Por tanto el sujeto debe señalar después de cada ítem la dirección de los síntomas, considerando que " +3 " correspondería a que el deportista considera que la sensación a la que se refiere en ese ítem le resulta muy facilitadora, mientras el "-3" supondría un gran obstáculo en el camino hacia un buen rendimiento. En algunos estudios se concede más importancia a la subescala de "dirección", ya que hay autores que afirman que tiene un mayor valor predictivo que la de intensidad, (Jones \& Hanton, 1996; 2001; Santos-Rosa, 2003).

Cox et al. (2003) realizaron un estudio con el propósito de revisar la estructura del CSAI-2, decidiendo tras el mismo, la eliminación de 10 ítems, dando lugar a la versión reducida CSAI-2R (Revised Competitive State Anxiety Inventory-2). Recientemente Andrade et al. (2007) realizaron la traducción de esta escala y analizaron sus propiedades psicométricas con una muestra de 149 deportistas españoles de diferentes disciplinas, concluyendo que esta versión cumple con las propiedades adecuadas, y sugiriendo que se realicen otros estudios con deportistas de diferentes niveles en nuestro país, con el fin de contrastar los resultados. 


\section{2.- Objetivo e hipótesis}

A la vista de la importancia que tiene la ansiedad estado precompetitiva en el rendimiento deportivo y de las características de la competición en judo, se pretende analizar la validez y fiabilidad del CSAI-2 en una muestra de judocas españoles de competición, y analizar si existen diferencias en la ansiedad de dichos judocas en función de su edad, género y nivel deportivo.

A partir de lo teorizado y de los estudios previos, planteamos la hipótesis de que los judocas con más edad, sobre la base de su mayor experiencia, presentan menores niveles de ansiedad cognitiva y somática (intensidad) que los jóvenes, y mayores niveles de autoconfianza intensidad y dirección. Además, los de más edad interpretarán de forma más positiva los síntomas de su ansiedad somática y cognitiva. Por otro lado, los judocas de mayor nivel deportivo presentarán una mayor autoconfianza que los de menor nivel. Finalmente creemos que se encontrarán diferencias en cuanto al género, presentando las judokas menores niveles de autoconfianza.

\section{3.- Método}

\section{1.- Muestra y procedimiento}

La muestra estuvo compuesta por 128 deportistas procedentes de una población de judocas españoles de competición, entre los que se encontraban representantes de todas las comunidades autónomas de nivel regional, nacional e internacional. Del total de los participantes un 53.1\% eran chicos y un $46.9 \%$ chicas. El rango de edad de los judocas estaba entre los 12 y los 35 años, y fueron divididos en dos grupos de modo que aquellos en edad infantil y cadete (entre 13 y 17 años) formaron parte del grupo "jóvenes" (57,4\%) mientras los de categoría junior, sub 23 y senior (17 años o más) compusieron el grupo de "adultos" (45,3\%). Por otro lado 57 deportistas pertenecían a los equipos nacionales de sus correspondientes categorías de edad, componiendo el grupo de "equipo nacional" mientras el resto (71 judocas) formaron parte del grupo "amateurs".

Todos los judocas de la muestra fueron informados de los propósitos del estudio y participaron de forma voluntaria, firmando un consentimiento informado que en el caso de los menores de edad se administró a los padres o tutores. Los participantes cumplimentaron el cuestionario la noche antes de la competición, a fin de no interferir en los intereses de los deportistas cuyo horario el día de la competición está ausente de huecos, desde que se levantan y asisten al pesaje, hasta que compiten. Los equipos nacionales fueron medidos en torneos internacionales, y los amateurs en campeonatos autonómicos y nacionales. Así, los judocas del equipo nacional senior fueron medidos en las World Cup de Austria (masculino) y Hungría (femenino) en 2007, torneos clasificatorios para los Juegos Olímpicos de Beijing 2008. Los judocas del equipo nacional infantil y cadete fueron medidos en el Campeonato de Europa de 2007, y los amateurs en campeonatos de nivel regional y nacional (Autonómico Senior de Valencia 2007, Fases de Sector Este y Centro sub 23 de 2008, Fase final del Campeonato de España sub 23 de 2008 y Campeonatos de España Infantil y Cadete de 2008). Dichos campeonatos supondrían un reto en función del nivel del deportista, ya que probablemente para un amateur, la dificultad que supone una fase de sector clasificatoria para el Campeonato de España es tan grande como la que supone para un judoca del equipo nacional un torneo internacional.

El difícil acceso a gran parte de los judocas, de diferentes procedencias del territorio nacional y de alto nivel deportivo en gran parte, hizo que se administrara el cuestionario en tan sólo una ocasión. No obstante se conocen estudios previos que han arrojado información sobre la ansiedad estado precompetitiva empleando esta metodología con judocas españoles (Hernández et al., 2008) o recientemente en el estudio realizado con gimnastas griegas (Tsopani et al., 2011).

\section{2.- Instrumentos de medida}

\section{Medida de la intensidad de la ansiedad estado previa a la competición}

Para medir la intensidad de la ansiedad estado precompetitiva se empleó una escala que ha sido diseñada con el fin de medir la ansiedad precompetitiva en el ámbito deportivo. La Competitive State Anxiety Inventory-2 (CSAI-2) de Martens et al. (1990b), validada al español por 
Barbero y Pérez-Llantada (1999) y Pozo (2007). Este cuestionario está compuesto por 3 factores (ansiedad somática, ansiedad cognitiva y autoconfianza) que se agrupan en 17 ítems en una escala Likert que va de 1 (nada) a 4 (mucho). Los participantes reflejan el estado de acuerdo o desacuerdo con las afirmaciones que versan sobre cómo se encuentran en ese momento.

La pregunta inicial que encabeza el cuestionario es "Indica cómo te sientes en este mismo momento..." a la cual le siguen los ítems relacionados con cada uno de los tres factores mencionados anteriormente. Así un ítem que mide la intensidad de la ansiedad somática sería, por ejemplo, "Mi corazón está acelerado", un ítem que mide la intensidad de la ansiedad cognitiva sería "En esta competición, me preocupa no ser capaz de hacerlo tan bien como yo sé que puedo" y de medida de la autoconfianza sería "Estoy seguro de poder superar este desafío".

El análisis de la consistencia interna del cuestionario desveló valores aceptables para los tres factores, ansiedad somática (.80), ansiedad cognitiva (.72), y autoconfianza (.73).

\section{Medida de la dirección de la ansiedad estado previa a la competición.}

Para medir la dirección de la ansiedad estado precompetitiva se empleó también la Competitive State Anxiety Inventory-2 (CSAI-2) de Martens et al. (1990b), traducida al español por Barbero y Pérez-Llantada (1999). Lo novedoso de esta escala con respecto a otras anteriores es que contempla la direccionalidad de la ansiedad. Por ello existe una subescala anexa a la de la intensidad de la ansiedad donde el sujeto debe manifestar en un rango de respuesta que va desde -3 (me perjudicará mucho) hasta +3 (me beneficiará mucho), si cree que la sensación manifestada en el ítem de la escala de intensidad le perjudicará o beneficiará en su rendimiento respecto a la competición a la que se enfrentará.

Los estudios de validez y fiabilidad realizados en castellano (Cervelló et al., 2002) de la subescala de direccionalidad encuentran alphas $>.70$. El análisis de consistencia interna del cuestionario desveló valores alpha aceptables para los tres factores. Así, el coeficiente alpha para los ítems del factor ansiedad somática dirección obtuvo un valor de .84, el de ansiedad cognitiva dirección .70, y el de autoconfianza dirección 83.

\section{4.- Resultados}

Se presentan a continuación los resultados del análisis factorial del instrumento utilizado para medir la ansiedad precompetitiva, los estadísticos descriptivos, media y desviación típica de los factores y el análisis de consistencia interna de los cuestionarios así como los análisis de varianza (2x2x2) para comprobar las diferencias entre grupos en función del género, nivel deportivo y categoría de edad.

\section{1.- Análisis de validez, fiabilidad y estadísticos descriptivos de los instrumentos}

Se realizó un análisis factorial exploratorio con rotación Varimax del CSAI-2 en su subescala Intensidad, que demostró la existencia de tres factores denominados ansiedad somática (6 ítems), ansiedad cognitiva (5 ítems) y autoconfianza (5 ítems), con autovalores mayores a 1.00 (3.03 para el factor ansiedad somática, 2.74 para el factor autoconfianza y 2.74 para el factor ansiedad cognitiva). Se eliminó el ítem 4 de la escala original, "mi cuerpo está tenso", porque saturaba en un factor diferente al que debiera teóricamente y se requirió un peso mínimo de .40 para que la variable pudiese ser considerada como importante (Carretero-Dios y Pérez, 2005) (Tabla 1).

De igual modo se realizó un análisis factorial para determinar la estructura de la escala CSAI-2 (Competitive State Anxiety Inventory-2) en su subescala de Dirección, de Martens et al. (1990), que mide la dirección de la ansiedad somática, ansiedad cognitiva y autoconfianza. También se requirió un peso mínimo de .40 (Carretero-Dios \& Pérez, 2005) para que la variable pudiese ser considerada como importante, demostrándose la existencia de tres factores denominados ansiedad somática dirección (7 ítems), ansiedad cognitiva dirección (5 ítems) y autoconfianza dirección (5 ítems) con autovalores mayores a 1.00 (3.51 para el factor ansiedad somática dirección, 3.10 para el factor autoconfianza dirección y 2.61 para el factor ansiedad cognitiva dirección) (Tabla 2). 
Tabla 1

Análisis factorial con rotación Varimax del CSAI-2 Intensidad sin el ítem 4

\begin{tabular}{|c|c|c|c|}
\hline \multirow[b]{2}{*}{ "Indica cómo te sientes en este mismo momento..." } & \multicolumn{3}{|c|}{ Intensidad de Ansiedad Estado } \\
\hline & Somática & Autoconfianza & Cognitiva \\
\hline 6. Siento tensión (nervios) en mi estómago & .825 & & \\
\hline 12. Siento un nudo en el estómago & .795 & & \\
\hline 9. Mi corazón está acelerado & .769 & & \\
\hline 15. Mis manos están sudorosas & 637 & & \\
\hline 17. Mi cuerpo está agarrotado & .588 & & \\
\hline 1. Me siento muy nervioso & 463 & & \\
\hline 7. Estoy seguro de poder superar este desafío & & .825 & \\
\hline 10. Estoy seguro de hacerlo bien & & .817 & \\
\hline 3. Confío en mí mismo & & .748 & \\
\hline $\begin{array}{l}\text { 13. Estoy confiado porque soy capaz de imaginarme consiguiendo } \\
\text { mis objetivos }\end{array}$ & & .630 & \\
\hline 16. Confío en no venirme abajo por la presión & & .524 & \\
\hline 11. Me preocupa hacerlo mal & & & .817 \\
\hline 5. Me preocupa perder & & & .755 \\
\hline $\begin{array}{l}\text { 2. En esta competición, me preocupa no ser capaz de hacerlo tan } \\
\text { bien como yo sé que puedo }\end{array}$ & & & 622 \\
\hline 14. Me preocupa que otros critiquen mi rendimiento & & & .598 \\
\hline 8. Me preocupa como actuaré bajo presión & & & .529 \\
\hline Autovalores & 3.03 & 2.74 & 2.74 \\
\hline Porcentaje de Varianza & 18.94 & 17.18 & 17.15 \\
\hline
\end{tabular}

Tabla 2

Análisis factorial con rotación Varimax del CSAI-2 Dirección

\begin{tabular}{|c|c|c|c|}
\hline \multirow{2}{*}{$\begin{array}{l}\text { "Indica cómo te sientes en este mismo momento, y si crees } \\
\text { que este estado te beneficiará o perjudicará..." }\end{array}$} & \multicolumn{3}{|c|}{ Dirección de la Ansiedad } \\
\hline & Somática & Autoconfianza & Cognitiva \\
\hline 12. Siento un nudo en el estómago. & .828 & & \\
\hline 15. Mis manos están sudorosas. & 817 & & \\
\hline 6. Siento tensión (nervios) en mi estómago. & .752 & & \\
\hline 17. Mi cuerpo está agarrotado. & .642 & & \\
\hline 4. Mi cuerpo está tenso. & .598 & & \\
\hline 1. Me siento muy nervioso. & .532 & & \\
\hline 9. Mi corazón está acelerado. & .517 & & \\
\hline 7. Estoy seguro de poder superar este desafío. & & .853 & \\
\hline 10. Estoy seguro de hacerlo bien. & & .818 & \\
\hline $\begin{array}{l}\text { 13. Estoy confiado porque soy capaz de imaginarme consiguiendo } \\
\text { mis objetivos. }\end{array}$ & & .789 & \\
\hline 3. Confío en mí mismo. & & .757 & \\
\hline 16. Confío en no venirme abajo por la presión. & & .513 & \\
\hline 11. Me preocupa hacerlo mal. & & & .809 \\
\hline 5. Me preocupa perder. & & & 689 \\
\hline $\begin{array}{l}\text { 2. En esta competición, me preocupa no ser capaz de hacerlo tan } \\
\text { bien como yo sé que puedo. }\end{array}$ & & & .606 \\
\hline 8. Me preocupa como actuaré bajo presión. & & & .514 \\
\hline 14. Me preocupa que otros critiquen mi rendimiento. & & & .420 \\
\hline Autovalores & 3.51 & 3.10 & 2.61 \\
\hline Porcentaje de Varianza & 20.66 & 18.26 & 15.39 \\
\hline
\end{tabular}

A continuación se presentan los estadísticos descriptivos resultantes de la estructura factorial hallada en el presente estudio, media y desviación típica, de la escala empleada para medir la ansiedad estado antes de la competición, además de la consistencia interna determinada a través del coeficiente alpha (Tabla 3). 
Tabla 3

Descriptivos y coeficientes de consistencia interna de la escala CSAI-2

\begin{tabular}{lccc}
\hline Ansiedad Estado Precompetitiva & Alpha & $\boldsymbol{M}$ & $\boldsymbol{D T}$ \\
\hline A. Somática (Intensidad) & .80 & 2.0459 & .62948 \\
A. Cognitiva (Intensidad) & .72 & 2.4238 & .70182 \\
Autoconfianza (Intensidad) & .73 & 3.1524 & .57713 \\
A. Somática (Dirección) & .84 & .0925 & 1.06096 \\
A. Cognitiva (Dirección) & .70 & -.2967 & 1.06863 \\
Autoconfianza (Dirección) & .83 & 1.3607 & 1.25036 \\
\hline
\end{tabular}

En torno a la intensidad de la ansiedad los resultados muestran que los judocas contemplados en la muestra presentan medias altas en el factor autoconfianza, algo más moderado con los ítems del factor ansiedad cognitiva, y más bajo con los del factor ansiedad somática, considerando que el rango de respuesta es de 1 a 4 . En cuanto a la dirección de la ansiedad los resultados muestran que los participantes tienen mayor grado de acuerdo con las afirmaciones contenidas en los ítems del factor autoconfianza (dirección), algo más moderado con los ítems del factor ansiedad somática (dirección), y más bajo con los del factor ansiedad cognitiva (dirección), considerando que el rango de respuesta es de $-3 \mathrm{a}+3$.

Por otro lado, el análisis de consistencia interna del cuestionario en sus dos subescalas, determinado a través del coeficiente alpha, muestra para todos los factores una alta fiabilidad (alpha $\geq .70$ ) con puntuaciones que oscilan entre .70 (como valor mínimo) para la medida de la dimensión ansiedad cognitiva (dirección) y de .84 (como valor máximo) para la medida de la ansiedad somática (dirección).

\section{2.- Análisis de las diferencias entre grupos}

Para ver las diferencias en la ansiedad estado precompetitiva de los judocas que componen la muestra en función del género, nivel deportivo y categoría de edad se han llevado a cabo diseños factoriales multivariados $(2 \times 2 \times 2)$.

Diferencias en función del género, nivel deportivo y categoría de edad en la intensidad de la ansiedad estado precompetitiva

Se realizó un análisis de varianza múltiple $(2 \times 2 \times 2)$ entre género, categoría de edad y nivel deportivo, apreciándose la existencia de diferencias significativas en las variables estudiadas para el nivel deportivo (Tabla 4)

Tabla 4

Análisis de varianza múltiple $(2 \times 2 \times 2)$ entre género por nivel deportivo por categoría

\begin{tabular}{lccc}
\hline Fuente & Lambda de Wilks & $\boldsymbol{F}$ & $\boldsymbol{p}$ \\
\hline Categoría & .966 & .990 & .416 \\
Nivel Deportivo & .915 & 2.635 & $.038^{*}$ \\
Género & .970 & .875 & .481 \\
Categoría x Nivel Deportivo & .982 & .516 & .724 \\
Categoría x Género & .975 & .738 & .568 \\
Nivel Deportivo x Género & .961 & 1.166 & .330 \\
Categoría x Nivel Deportivo x Género & .926 & 2.282 & .065 \\
\hline
\end{tabular}

$p<.05^{*}$ 
Las pruebas de los efectos inter-sujetos por nivel deportivo llevadas a cabo a continuación (Tabla 5) indican que los judocas de equipo nacional y los amateurs difieren significativamente en la intensidad de la autoconfianza, $F=7.03, p \leq .05$ de forma que los judocas del equipo nacional tienen mayor autoconfianza $(\mathrm{M}=3.32)$ que los amateurs (3.04).

Tabla 5

Análisis de la varianza para el Nivel Deportivo

\begin{tabular}{lccccc}
\hline & & & & \multicolumn{2}{c}{ M } \\
\cline { 6 - 7 } Variables & MC & F & p & E. Nacional & Amateurs \\
\hline Ansiedad Estado Intensidad & & & & & \\
Somática & .61 & 1.505 & .222 & 1.97 & 2.12 \\
Cognitiva & .76 & 1.513 & .221 & 2.35 & 2.52 \\
Autoconfianza & 2.11 & 7.037 & $.009^{*}$ & 3.32 & 3.04 \\
\hline$p<.05^{*}$ & & & &
\end{tabular}

Diferencias en función del género, nivel deportivo y categoría de edad en la dirección de la ansiedad estado precompetitiva

Se realizó un análisis de varianza múltiple $(2 \times 2 \times 2)$ entre género, nivel deportivo y categoría de edad, apreciándose la existencia de diferencias significativas en las variables estudiadas para la categoría de edad (Tabla 6)

Tabla 6

Análisis de varianza múltiple (2×2×2) entre género por nivel deportivo por categoría

\begin{tabular}{lccc}
\hline Fuente & Lambda de Wilks & $\boldsymbol{F}$ & $\boldsymbol{p}$ \\
\hline Categoría & .909 & 3.726 & $.013^{*}$ \\
Nivel Deportivo & .958 & 1.619 & .189 \\
Género & .946 & 2.115 & .102 \\
Categoría x Nivel Deportivo & .989 & .417 & .741 \\
Categoría x Género & .963 & 1.404 & .245 \\
Nivel Deportivo x Género & .971 & 1.094 & .355 \\
Categoría x Nivel Deportivo x Género & .966 & 1.319 & .272 \\
\hline$p<.05 *$ & & &
\end{tabular}

Las pruebas de los efectos inter-sujetos por categoría de edad llevadas a cabo a continuación (Tabla 7) indican que los jóvenes y los adultos difieren significativamente tanto en la ansiedad somática (dirección), $F=10.33, p \leq .05$, como en la ansiedad cognitiva (dirección), $F=$ $6.08, p \leq .05$ de forma que los adultos consideran que sus niveles de ansiedad somática favorecerán su rendimiento $(M=.42)$ en mayor medida que los jóvenes $(-.22)$, y consideran sus niveles de ansiedad cognitiva $(M=-.02)$ menos negativas que los jóvenes $(-.53)$.

Tabla 7

Análisis de la varianza para la categoría de edad

\begin{tabular}{lccccc}
\hline & & & & \multicolumn{2}{c}{$\boldsymbol{M}$} \\
\cline { 5 - 6 } Variables & $\boldsymbol{M C}$ & $\boldsymbol{F}$ & $\boldsymbol{p}$ & Jóvenes & Adultos \\
\hline Ansiedad Estado Dirección & & & & & \\
Somática & 10.71 & 10.336 & $.002^{*}$ & -.22 & .42 \\
Cognitiva & 6.72 & 6.088 & $.015^{*}$ & -.53 & -.02 \\
Autoconfianza & 2.82 & 1.937 & .167 & 1.18 & 1.51 \\
\hline
\end{tabular}

$p<.05^{*}$ 


\section{5.- Discusión}

El objetivo de este trabajo fue comprobar las propiedades psicométricas del CSAI-2 en una muestra de judocas españoles, con el fin de constatar lo adecuado de su uso para medir ansiedad estado precompetitiva en este colectivo. Tal y como sucediera en estudios previos con deportistas españoles (Barbero et al., 1999; Pozo, 2007) la escala ha mostrado unas propiedades psicométricas aceptables, si bien en nuestra muestra ha tenido que ser eliminado un ítem de la subescala Intensidad, ya que saturaba en un factor diferente al que debiera teóricamente.

Además, en la presente investigación se pretendió analizar las posibles diferencias existentes en la ansiedad estado precompetitiva de judocas en función a su nivel deportivo, edad y género. Para eso, formaron parte de la muestra judocas de competición de diferentes niveles deportivos, incluyendo un gran número de deportistas del equipo nacional. Entendiendo que la experiencia es parte fundamental en el proceso de aprendizaje y control del estado psicológico previo a las competiciones, han participado en la investigación deportistas de edades muy dispares dentro del rango que permite la disciplina del judo, ya que la competición empieza a ser concebida como tal desde la categoría infantil, donde ya se disputan campeonatos de España, hasta los 34 o 35 años que es la edad máxima hasta la que normalmente los judocas pueden seguir compitiendo.

Se esperaba que se manifestaran diferencias en la ansiedad estado en función de su edad, de modo que los adultos experimentaran niveles más bajos, que los jóvenes, en la intensidad de la ansiedad somática y cognitiva, como ya sucediera en otras investigaciones (Hanton et al., 2008; Hernández et al., 2008; Kuuseok \& Ratassepp, 2001; Mellalieu et al.,2004), además de hacer una interpretación más positiva de los síntomas (Cerin, Szabo, Hunt, \& Williams, 2000; Hanton \& Jones, 1999). Se hipotetizó también que los adultos mostrarían además puntuaciones más altas en las dos subescalas (intensidad y dirección) de la autoconfianza, por entenderse esta como otra consecuencia lógica adaptativa ligada a una mayor experiencia. Cuando se plantearon las hipótesis se entendió que los judocas de más edad del presente estudio eran más expertos, aún a sabiendas del riesgo que supone hacer esta asociación de ideas, y que los años de edad o de práctica, no siempre son sinónimos de una mayor experiencia, tal y como se describe en Hanton et al. (2009).

A tenor de los resultados de esta investigación se puede afirmar que la hipótesis se cumple parcialmente, de modo que no se han encontrado las diferencias esperadas en la intensidad de la ansiedad, pero sí en la interpretación que los judocas hacen de los síntomas de la ansiedad tanto somática como cognitiva. Parece que los judocas adultos del estudio sienten niveles similares de ansiedad que los jóvenes, pero consideran que dichos síntomas serán más positivos (en el caso de la ansiedad somática) o menos negativos (en la ansiedad cognitiva) para su rendimiento próximo. Tampoco se apreciaron diferencias en ninguna de las dos subescalas de la autoconfianza en función de la edad de los sujetos. El hecho de que no se hayan confirmado las diferencias esperadas en la intensidad de ansiedad estado, podría deberse al hecho ya mencionado de entender que los judocas del grupo adulto son más expertos que los del otro grupo, sabiendo que no se ha tenido en cuenta el número de competiciones, o tipo de práctica que han realizado los mismos durante el transcurso de su carrera deportiva, ni siquiera, se ha indagado sobre los años de práctica de los deportistas. Aún así, se han encontrado diferencias en la interpretación de los síntomas, por lo que podría resultar oportuno realizar un estudio futuro indagando de forma más concisa sobre la ansiedad estado precompetitiva de los judocas, pero esta vez analizando la experiencia desde el prisma de una práctica reflexiva, o deliberada, que ofrezca la posibilidad de reducir el margen de error en lo que a la experiencia se refiere. En esta línea Cepeda et al. (2011) han analizado aspectos referentes a la práctica deliberada de judocas españoles de competición, concluyendo que judocas de diferentes niveles entienden como muy importante el entrenamiento psicológico para su rendimiento, además de entender que todas las variables importantes para rendir hay que entrenarlas en condiciones de concentración y pertinencia (aspectos relativos a la práctica deliberada).

En cualquier caso, conviene recordar el estudio realizado con judocas griegos (Nasioudis et al., 2005), donde se hace referencia a los cambios que se producen en el nivel de ansiedad estado precompetitiva en función de que el deportista arrastre o no problemas de peso. En este estudio no se ha controlado esta variable, lo que conduce a pensar que el motivo por el que no se han obtenido algunos resultados esperados, se pueda deber a que algunos de los judocas de este estudio, asistieran a las competiciones donde fueron testados con problemas de peso y otros no. 
También se hipotetizó que el nivel deportivo marcaría diferencias entre grupos en las diferentes dimensiones de la ansiedad estado medidas en situación precompetitiva, tal y como sucediera en algunos estudios realizados previamente con deportistas (Hanton \& Connaughton, 2002; Hanton et al., 2003; Jones et al., 1994; Jones \& Swain, 1992; Perry \& Williams, 1998), donde una de las variables más importantes en la forma de interpretar los síntomas de la ansiedad era precisamente esa. La hipótesis se cumple parcialmente, ya que solo se han encontrado diferencias en la intensidad de la autoconfianza entre los judocas del equipo nacional y los amateurs. Parece que los judocas del equipo nacional confían más en sus posibilidades a la hora de enfrentarse a la competición, al igual que sucediera en el estudio con tenistas (Covassin \& Pero, 2004) en el que los ganadores experimentaban mayores niveles de autoconfianza, previa al partido, que los perdedores. Probablemente, los deportistas de mayor nivel deportivo, tengan más experiencia en las situaciones competitivas, lo que hace que sepan interpretar mejor la competencia que tienen sobre el reto al que se van a enfrentar, teniendo en cuenta que la autoconfianza es una evaluación cognitiva de las propias capacidades para afrontar un reto y hace referencia a una evaluación subjetiva de la competencia. Los resultados obtenidos en la presente investigación se asemejan a los presentados por Perry y Williams (1998), en una investigación llevada a cabo con tenistas, donde el grupo de mayor nivel deportivo mostró mayores niveles de autoconfianza, pero no se encontraron diferencias en la ansiedad somática.

Finalmente, considerando los resultados obtenidos en anteriores estudios (Abrahamsen, Roberts, \& Pensgaard, 2008; Brunet \& Sabiston, 2008) donde los chicos mostraran menores niveles de ansiedad que las chicas, se esperaba que pudieran hallarse diferencias en la ansiedad en función del género. En este caso, no sucedió así, como ya ocurriera en el estudio realizado con judocas de élite coreanos (Woo, 1996), donde tampoco se encontraron diferencias en el nivel de ansiedad en función del género. Hay que tener en cuenta que en los estudios señalados donde sí se encontraron diferencias (Abrahamsen et al., 2008; Brunet \& Sabiston, 2008), se midió ansiedad social física, que si bien es un tipo de ansiedad, no es exactamente igual que la competitiva. Tampoco encontramos diferencias en la autoconfianza entre chicos y chicas, al contrario de lo que sucediera en un estudio antiguo realizado con judocas brasileños (Moraes, 1987), donde los chicos mostraron niveles más elevados que las chicas en esa variable. Parece por tanto que los judocas de nuestro país no experimentan más ansiedad o menos en función de que pertenezcan a uno u otro género.

\section{6.- Conclusiones}

A modo de conclusiones, podemos decir que el CSAI-2 ha presentado unas propiedades psicométricas adecuadas en una muestra de judocas de competición españoles. Además, los judocas adultos interpretan los síntomas de sus niveles de ansiedad somática y cognitiva de forma más positiva que los judocas de menos edad, mientras el nivel deportivo a supuesto diferencias en lo que a la ansiedad estado se refiere, de modo que los deportistas del equipo nacional confían más en sus posibilidades a la hora de enfrentarse al reto de la competición. Finalmente el género no ha supuesto diferencias ni en la intensidad ni en la dirección de la ansiedad en el presente estudio.

Por último, destacar cómo a pesar de las aportaciones del estudio realizado, este presenta algunas limitaciones metodológicas entre las que podemos destacar: el momento de aplicación del cuestionario empleado para medir la ansiedad precompetitiva, que si bien habría sido más acertado administrarlo instantes antes de la competición, ha tenido que hacerse la noche antes de la misma por motivos de estructura temporal en la organización de los diferentes torneos (ya explicada en el procedimiento). En segunda instancia, se podría considerar más apropiado el empleo de la escala reducida del CSAI-2, ya que existe otra escala más actualizada del instrumento, CSAI-2R (Cox et al., 2003) que cumple con las propiedades psicométricas adecuadas para medir la variable objeto de nuestro estudio en deportistas españoles (Andrade et al., 2007).

Futuras investigaciones podrían analizar si realmente existen diferencias en los niveles de ansiedad estado precompetitiva en los judocas en función de que tengan problemas de peso, así como ahondar por medio de trabajos experimentales en la eficacia de algunas estrategias que pudieran llevar a cabo los entrenadores para reducir los niveles de ansiedad cognitiva y somática de los judocas, aumentando su autoconfianza. Sería interesante medir la ansiedad estado 
precompetitiva de los judocas españoles de competición con la versión reducida del CSAI-2, tal y cómo recomiendan Andrade et al. (2007), para ver si se comporta igual de bien que la versión inicial. Además sería importante realizar estudios que analizaran la relación de ansiedad estado precompetitiva con el rendimiento medido de forma objetiva y ver la relación que guarda con otras variables como el estado de ánimo precompetitivo o el estado de flow experimentado por los deportistas en una competición.

\section{7.- Aplicaciones prácticas}

De los resultados obtenidos se pueden extrapolar algunas aplicaciones al campo práctico, de modo que los entrenadores podrían ayudar a los deportistas que están bajo su tutela, a interpretar los síntomas ansiosos típicos de una situación precompetitiva, planificando el número de competiciones necesarias para que se produzca una adaptación y dialogando con ellos sobre lo natural que es sentirse algo ansiosos. La recomendación que desde estas líneas se hace, es trabajar esta variable del mismo modo que se trabajan los aspectos condicionales, técnicos o tácticos, diseñando ciclos en el tiempo y estrategias para la mejora de dicha capacidad (Wolframm \& Micklewright, 2011). Parece que los judocas de más nivel se sienten más capacitados a la hora de afrontar las competiciones que aquellos que son de un menor nivel, por ello entendemos que aumentar el dominio de los deportistas va a hacer que esos niveles de autoconfianza aumenten.

Por otro lado, el empleo de algunas estrategias de afrontamiento los días previos a la competición, tales como autoinstrucciones, visualización, o estrategias de afrontamiento del estrés focalizando la atención en los aspectos que se pueden controlar, se han mostrado muy útiles en anteriores estudios con deportistas de diferentes niveles y modalidades deportivas (Bertollo, Saltarelli, \& Robazza, 2009; Gould et al., 1993; Hatzigeorgiadis et al., 2009; Mamassis \& Dogamis, 2004) y podrían ayudar a aumentar la autoconfianza de los judocas de cara a la competición. Por ejemplo, los días previos al torneo, los técnicos podrían centrarse en reforzar las acciones que el deportista hace bien, destacando los aspectos positivos de ejecución motriz o acciones favorecedoras de un estado emocional positivo, sin incidir en aquellas técnicas o secuencias tácticas que el deportista aún no domina. Esta estrategia podría ser más acertada que la de intentar solucionar de forma insistente las carencias del competidor, contribuyendo a que este se centre en los aspectos que le ayuden a pensar que está preparado para el reto al que se enfrentará. Este hecho puede reducir las probabilidades de que el deportista alcance niveles demasiado elevados de ansiedad, potenciando a la vez su autoconfianza y requiriendo por tanto una evaluación continua y periódica de los niveles de ansiedad y autoconfianza en los momentos críticos antes de la competición. Otra estrategia para aumentar la autoconfianza podría consistir en hacer entrenamientos en los que el deportista acumule muchas proyecciones y acciones exitosas, por las características de la tarea (e.g., nague-komis), o del rival (nivel, edad, o peso). En esa línea, podría ser muy adecuada la visualización de filmaciones en torneos anteriores en las que el judoca sale airoso, de forma que se ve capaz de repetirlo porque ya lo ha hecho bien antes. Evitar hablar de las virtudes o del currículum de los rivales que encontrará el deportista, haciendo hincapié en que él solo tiene que hacer lo que sabe, hará que el deportista se sienta con más capacidad para controlar la tarea futura. Además, realizar un estudio de rivales y trabajarlo con la antelación suficiente puede hacer que se rebaje el grado de incertidumbre, y así los niveles de ansiedad cognitiva y somática, aumentándose la autoconfianza, ya que el deportista sabrá que ya ha solucionado anteriormente (en los entrenamientos) los problemas a los que se va a enfrentar. Todas estas consideraciones podrían ser beneficiosas en el fomento de la autoconfianza, con las conocidas ventajas que esto conlleva al mediatizar las relaciones entre ansiedad y rendimiento (López Torres et al., 2007).

\section{Referencias}

Abrahamsen, F.A.; Roberts, G.C.; Pensgaard, A.M. (2008). Achievement goals and gender effects on multidimensional anxiety in national elite sport. Psychology of Sport and Exercise, 9(4), 449464.

Andrade, E.M.; Lois, G.; Arce, C. (2007). Propiedades psicométricas de la versión española del inventario de ansiedad competitiva CSAI-2R en deportistas. Psicothema, 19, 150-155. 
Bandura, A. (1982). Self-efficacy. Mechanism in human agency. American Psychologist, 377, 122147.

Barbero García, M.I. y Pérez-Llantada, M.C. (1999). Cuestionarios en psicología del deporte. En A. López de la Llave, M. C. Pérez-Llantada y J. M. Buceta (Eds), Investigaciones breves en psicología del deporte (pp. 40-45). Madrid: Dykinson.

Bertollo, M; Saltarelli, B; Robazza, C. (2009). Mental preparation strategies of elite modern pentathletes. Psychology of Sport and Exercise, 10, 244-254.

Borkoveck, T.D. (1976). Psichologycal and cognitive process in the regulation of anxiety. En G. Schwart, y D. Shapiro (Eds), Consciousness and self-regulation: Advances in research, 1 (pp. 261-312). New York: Plemen Press.

Brandao, R. F. (1995). Ansiedade em atletas. Movimento, 1, 24-27

Brunet, J. y Sabiston, C.M. (2008). Social physique anxiety and physical activity: A selfdetermination theory perspective. Psychology of Sport and Exercise, doi: 10.1016/j.psychsport.2008.11.002.

Burton, D. (1988). Do anxious swimmers swim slower? Reexamining the elusive anxietyperformance relationship. Journal of Sport and Exercise Psychology, 10, 45-61.

Carretero-Dios, H y Pérez, C. (2005). Normas para el desarrollo y revisión de estudios instrumentales. International Journal of Clinical and Health Psychology, 5(3), 521-551.

Cepeda, M.; Montero, C.; García, L.; León, B.; Iglesias, D. (2011). La percepción de judocas de distinto nivel de pericia respecto a las actividades de entrenamiento específicas que contribuyen al rendimiento. Archivos de Medicina del Deporte, 28(145), 257-264.

Cerin, E.; Szabo, A.; Hunt, N.; Williams, C. (2000). Temporal patterning of competitive emotions: A critical review. Journal of Sports Sciences, 18, 605-626.

Cervantes, J.L.; Rodas, G; Capdevila, L. (2009). Perfil psicofisiológico de rendimiento en nadadores basado en la variabilidad de la frecuencia cardíaca y en estados de ansiedad precompetitiva. Revista de Psicología del Deporte, 18(1), 37-52.

Cervelló, E.; Santos-Rosa, F.J.; Jiménez, R.; Nerea, A.; García, T. (2002). Motivación y ansiedad en jugadores de tenis. Revista Motricidad, 9, 141-161.

Cheng, W.-N. K.; Hardy, L.; Markland, D. (2009). Toward a three-dimensional conceptualization of performance anxiety: Rationale and initial measurement development. Psychology of Sport and Exercise, 10, 271-278.

Covassin, T. y Pero, S. (2004). The Relationship between Self-Confidence, Mood State and Anxiety among Collegiate Tennis Players. Journal of Sport Behavior, 27(3), 230-242.

Cox, R.H.; Martens, M.P.; Russell W.D. (2003). Measuring anxiety in athletics: The revised Competitive State Anxiety Inventory-2. Journal of Sport and Exercise Psychology, 25, 519-533.

Craft, L.L.; Magyar, T.M.; Becker, B.J.; Feltz, D.L. (2003). The relationship between the competitive state anxiety inventory-2 and sports performance: A meta-analysis. Journal of Sport and Exercise Psychology, 25, 44-65.

Davidson R.J. y Schwartz, G.E. (1976). The psicobiology of relaxation and related states: A multiprocess theory. En D. Mostovsky (Ed.), Behavioural control and modification of psychological activity (pp. 399-442). Englewood Cliffs, NJ: Prentice-Hall.

Eysenck, M.W. y Calvo, M.G. (1992). Anxiety and performance. The processing efficiency theory. Cognition and Emotion, 6, 409-434.

Filaire, E.; Sagnol, M.; Ferrand, C.; Maso, F.; Lac, G. (2001). Psychophysiological stress in judo during competitions. Journal of Sports Medicine and Physical Fitness, 41, 263-268.

Gould, D. (1987). Promoting positive sport experiences for children. In: M.J. Ash and J. May, Editors, Sport psychology: The psychological health of the athlete. New York: PMA Publishers.

Gould, D.; Eklund, R.C.; Jackson, S.A. (1993). Coping strategies used by U.S. Olympic wrestlers. Research Quarterly of Exercise and Sport, 64, 83-93.

Gould, D.; Greenleaf, C.; Krane, V. (2002). The arousal-athletic performance relationship: Current status and future directions. En T.S. Horn (Ed.), Advances in sport psychology (2nd ed.; pp. 207241). Champaign, IL: Human Kinetics.

Gould, D. y Krane, V. (1992). The arousal-athletic performance relationship: current status and future directions. En T. Horn (Ed). Advances in sport psychology (pp. 119-141). Champaign, IL: Human Kinetics. 
Gould, D.; Petlichkoff, L.; Weinberg, R.S. (1984). Antecedents of temporal changes in and relationships between CSAI-2 components. Journal of Sport Psychology, 6, 289-304.

Hammermeister, J. y Burton, D. (1995). Anxiety and the Ironman: Investigating the antecedents and consequences of endurance athletes' state anxiety. The Sport Psychologist, 9, 29-40.

Hanin, Y.L., (1980). A study of anxiety in sport. En W. P. Starub (Ed.), Sport Psychology: An Analysis of Athletic Behabior (pp. 236-249) Ithaca, NY: Movement Publications.

Hanin, Y.L. (1993). Optimal performance emotions in top athletes. En S. Serpa, J. Alves, V. Ferreira y A. Paula Brito (Eds.). Sport Psychology: An integrated approach. Proceedings of the VII World Congress of Sport Psychology (pp. 229-232). Lisboa.

Hanin, Y.L. (1997). Emotions and athletic performance: Individual zones of optimal functioning model. European Yearbook of Sport-Psychology, 1, 29-72.

Hanton, S.; Cropley, B.; Lee, S. (2009). Reflective practice, experience, and the interpretation of anxiety symptoms. Journal of Sports Sciences, 27(5), 517-533.

Hanton, S. y Connaughton, D. (2002). Perceived control of anxiety and its relationship to selfconfidence and performance. Research Quarterly for Exercise and Sport, 73, 87-97.

Hanton, S.; Evans, L.; Neil, R. (2003). Hardiness and the competitive trait anxiety response. Anxiety, Stress, and Coping, 16, 167-184.

Hanton, S. y Jones, G. (1999). The acquisition and development of cognitive skills and strategies: I. Making the butterflies fly in formation. The Sports Psychologist, 13, 4-21.

Hanton, S.; Mellieau, S.D.; Hall, R. (2004). Self-confidence and anxiety interpretation: A qualitative investigation. Psychology of Sport and Exercise, 5, 477-495.

Hanton, S.; Neil, R.; Mellalieu, S.D.; Fletcher, D. (2008). Competitive experience and performance status: An investigation into multidimensional anxiety and coping. European Journal of Sport Science, 8, 143-152.

Hardy, L. (1997). The Coleman Roberts Griffiths address: Three myths about applied consultancy work. Journal of Applied Sport Psychology, 9, 277-294.

Hardy, L. y Facey, J. (1987). The inverted-U hypothesis: A catastrophe for sport psychology. Paper presented at the meeting of the North American Society for the Psychology of Sport and Physical Activity, Vancouver, BC.

Hardy, L.; Jones, G.; Gould, D. (1996).Understanding psychological preparation in sport: theory and practice of elite performers. Chichester, U.K: Wiley.

Hatzigeorgiadis, A.; Zourbanos N.; Mpoumpaki, S.; Theodorakis, Y. (2009). Mechanisms underlying the self-talk - performance relationship: The effects of self-talk on self-confidence and anxiety. Psychology of Sport and Exercise, 10, 186-192.

Hernández, R.; Olmedilla, A.; Ortega, E. (2008) Ansiedad y autoconfianza de jóvenes judokas en situaciones competitivas de alta presión. Anállse Psicológica, 26(4), 689-696.

Jaenes, J.C. y Caracuel, J.C. (2005). Investigación acerca de la ansiedad precompetitiva. En Maratón. Preparación psicológica para el entrenamiento y la competición (pp. 83-99). Sevilla: Almuzara.

Jones, G. (1991). Recents developments and current issues in competitive state anxiety research. The Sport Psychologist, 4, 152-155.

Jones, G. (1995). More than just a game: research developments and issues in competitive anxiety in sport. British journal of psychology, 86, 4, 449-478.

Jones, G. y Hanton, S. (1996), Interpretation of competitive anxiety symptoms and goal attainment expectations. Journal of Sport y Exercise Psychology, 18, 144-157.

Jones, G. y Hanton, S. (2001). Pre-competitive feeling states and directional anxiety interpretations. Journal of Sports Sciences, 19, 385-395.

Jones, G.; Hanton, S.; Swain, A.B.J. (1994). Intensity and interpretation of anxiety symptoms in elite and non-elite sports performers. Personality and Individual Differences, 17, 657-663.

Jones, G. y Swain, A.B.J. (1992). Intensity and direction as dimensions of competitive state anxiety and relationships with competitiveness. Perceptual and Motor Skills, 74, 467-472.

Jones, G. y Swain, A.B.J. (1995). Predispositions to experience facilitating and debilitating anxiety in elite and non-elite performers. The Sport Psychologist, 9, 201-211.

Jones, G.; Swain, A.; Hardy, L. (1993). Intensity and direction dimensions of competitive state anxiety and relationships with performance. Journal of Sport Science, 11, 525-532. 
Kuuseok,-E y Ratassepp,-E. (2001). Pre-competitive state anxiety of young judo athletes. Sportlaste voistluseelse seisundi uurimine. Kehakultuuriteaduskonna-teadus-ja-oppemetoodiliste-toodekogumik-(Tartu) 92001, 110-115.

Landers, D.M. y Boutcher, S.H. (1986). Arousal performance relationship. En J.M. Williams (comp.), Applied sport psychology: personal growth to peak performance. Palo Alto, Ca.: Mayfield Publishing Company.

Liebert, R.M. y Morris, L.W. (1967). Cognitive and emotional components of tests anxiety: A distinction and some initial data. Psychologist Reports, 20, 975-978.

López-Torres, M.; Torregrosa, M.; Roca, J. (2007). Características del "Flow", ansiedad y estado emocional en relación con el rendimiento de deportistas de elite. Cuadernos de Psicología del Deporte, 7(1), 25-44.

Mamassis, G. y Dogamis, G. (2004). The effects of a mental training program on juniors precompetitive anxiety, self-confidence, and tennis performance. Journal of Applied of Sport Psychology, 16, 118-137.

Marahrens, L. y Keil, J.G. (2004). Training champions. An analysis of the phenomenon from the athlete's point of view. Zeitschriftfur Sportpsychologie, 11(3), 112-120.

Márquez, S. (2004). Ansiedad, estrés y deporte. Madrid: EOS.

Martens, R. (1977). Competitive State Anxiety Test. Champaign, IL: Human Kinetics.

Martens, R.; Vealey, R.S.; Burton, D (1990a). Competitive Anxiety in Sport. Champaign, IL: Human Kinetics.

Martens, R.; Burton, D.; Rivkin, F.; Simo, J. (1980). Reliability and validity of the Competitive State Anxiety Inventory (CSAI). En C. H. Nadeau, W. C. Halliwell, K. M. Newell, \& G. C. Roberts (Eds.), Psychology of Motor Behavior and Sport (pp.91-99). Champaign, IL: Human Kinetics.

Martens, R.; Burton, D.; Vealey, R.S.; Bump, L.A.; Smith, D.E. (1982). Competitive state anxiety inventory-2. Symposium conducted at the meeting of North American Society for the Psychology of Sport and Physical Activity (NASPSPA), College Park, MD.

Martens, R.; Burton, D.; Vealey, R.S.; Bump, L.A.; Smith, D.E. (1990b). Development and validation of the Competitive State Anxiety Inventory-2 (CSAI-2). En R. Martens. R.S. Vealey, y D. Burton, Competitive anxiety in sport (pp. 117-190). Champaign, IL: Human Kinetics.

Maynard, I.W. y Cotton, P.C. (1993). An investigation of two stress-management techniques in a field setting. The Sport Psychologist, 7, 375-387.

Maynard, I.W.; Hemmings, B.; Warwick-Evans, L. (1995). The effects of a somatic intervention strategy on competitive state anxiety and performance in semi-professional soccer players. The Sport Psychologist, 9, 51-64.

Maynard, I.W. y Howe, B.L. (1987). Interrelations of trait anxiety and state anxiety with games performance of rugby players. Perceptual and Motor Skills, 64, 599-602.

Maynard, I.W.; Smith, M.J.; Warwick-Evans, L. (1995). The effects of a cognitive intervention strategy on competitive state anxiety and performance in semiprofessional soccer players. Journal of Sport and Exercise Psychology, 17, 438-446.

Mellalieu, S.D.; Hanton, S.; O'brien, M. (2004). Intensity and direction of competitive anxiety as a function of sport type and experience. Scandinavian Journal of Medicine and Science in Sports, 14, 326-334.

Moraes, L.C. (1987). The relationship between anxiety and performance of Brasilian judokas. Unpublished master's thesis, Michigan State University.

Moya-Albiol, L.; Salvador, A.; Costa, R.; Martinez-Sanchis, S.; González-Bono, E.; Ricarte, J.; Arnedo, M. (2001). Psychophysiological responses to the stroop task after a maximal cycle ergometry in elite sportsmen and physically active subjects. International journal of psychophysiology, 40(1): 47-59.

Nasioudis, CH.; Kabitsi, A.; Kabitsis, CH.; Harahousou, Y.; Lailoglou, A. (2005). The Effect of Weighing on State Anxiety among Judo Players. Physical Training. http://ejmas.com/pt/2005pt/ptart Nasioudis 0805.html.

Perry, J.D. y Williams, J.M. (1998). Relationship of intensity and direction of competitive trait anxiety to skill level and gender in tennis. The Sport Psychologist, 12, 169-179.

Pozo, A. (2007). Intensidad y dirección de la ansiedad competitiva y expectativas de resultados en atletas y nadadores. Revista de Psicología del Deporte, 16(2), 137-150. 
Raudsepp, L. y Kais, K. (2002). The relationship between state anxiety and performance in beach voleyball players. Journal of Human Movement Studies, 43, 403-416.

Rodrigo, G.; Luisardo, M.; Pereira, G. (1990). Relationship between anxiety and performance in soccer players. International Journal of Sport Psychology, 21, 112-120.

Ruiz, M.C. y Hanin, Y.L. (2004a). Idiosyncratic description of anger states in skilled Spanish karate athletes: an application of the IZOF model. Revista de Psicología del Deporte, 13, 75-93.

Ruiz, M.C. y Hanin, Y.L. (2004b). Athlete's self perceptions of optimal states in karate: an application of the IZOF model. Revista de Psicología del Deporte, 13, 229-244.

Salvador, A.; Suay, F.; González-Bono, E.; Serrano, M.A. (2003). Anticipatory cortisol, testosterone and psychological responses to judo competition in young men. Psychoneuroendocrinology, 28, 364-375.

Santos-Rosa, F.J. (2003). Motivación, ansiedad y flow en jóvenes tenistas. Tesis doctoral. Cáceres: Universidad de Extremadura.

Smith, R.E. (2008). Avances en la Teoría Cognitivo-Social de la Personalidad: Aplicaciones a la Psicología del Deporte. Revista de Psicología del Deporte, 17(2), 253-276.

Smith, R.E.; Smoll, F.L.; Schutz, R.W. (1990). Measurements and correlates of sport-specific cognitive and somatic trait anxiety: the sport anxiety scale. Anxiety Research, 2, 263-280.

Spence, J.T. y Spence, K.W. (1966). The motivational components of manifest anxiety: Drive and drive stimuli. En C.D. Spielberger (Ed.), Anxiety and behavior (pp. 291-326). New York. Academic Press.

Spielberger, C.D. (1972). Conceptual and methodological issues in anxiety research. En C.D. Spielberger (Ed.), Anxiety: Current trends in theory and research (vol.2, pp. 23-49). New York: Academic Press.

Spielberger, C.D. y Díaz, R. (1975). IDARE Inventario de Ansiedad Estado-Rasgo. México: Manual Moderno.

Tsopani, D.; Dallas, G; Skordilis, E.K. (2011).Competitive state anxiety and performance in young female rhythmic gymnasts. Perceptual and Motor Skills, 112(2), 549-560.

Weinberg, R.S. y Gould, D. (2010). Fundamentos de Psicología del Deporte y del Ejercicio Físico (4⿳亠丷a ed.) Madrid: Editorial Médica Panamericana.

Weinberg, R. S. y Gould, D. (2003). Foundations of sport y exercise psychology (3 ${ }^{\text {rd }}$ ed.). Champaign, IL: Human Kinetics.

Wolframm, I.A. y Micklewright, D. (2011). The effect of a mental training program on state anxiety and competitive dressage performance. Journal of Veterinary Behavior, 6, 267-275.

Woo Han, M. (1996). Psychological profiles of Korean elite judoists. American Journal of Sports Medicine, 24(6), 67-71.

\section{Datos biográficos de los autores}

Carlos Montero Carretero es Licenciado en Ciencias de la Actividad Física y el Deporte, Doctor en Psicología, Maestro Entrenador Nacional de Judo, Cinturón Negro 5o DAN, Entrenador y Director Técnico del Club de Judo Miriam Blasco, Entrenador del Centro de Tecnificación Deportiva de Alicante, Doble Campeón de España Senior, Medalla de Bronce en Campeonato de Europa por Equipos y Bronce en Campeonato del Mundo Universitario. E-mail: cmontero@umh.es

Juan A. Moreno-Murcia es Licenciado en Educación Física y Doctor por la Universidad de Valencia. Acreditado Catedrático de Universidad y profesor en la Facultad de Ciencias Sociosanitarias de la Universidad Miguel Hernández de Elche. Es docente de la asignatura "Enseñanza de la actividad física y del deporte", así mismo, también es investigador del Grupo de Investigación en Comportamiento Motor y autor de numerosos libros y artículos en revistas científicas. Sus líneas de investigación se centran en el estudio de los procesos de enseñanza-aprendizaje de las actividades físico-deportivas. E-mail: j.moreno@umh.es

Inmaculada González Ponce es Licenciada en Ciencias de la Actividad Física y el Deporte por la Universidad de Extremadura. Tiene un Máster Universitario en Investigación en Ciencias Sociales y Jurídicas con Especialidad en Ciencias de la Actividad Física y el Deporte, así como un Máster Universitario en Formación del Profesorado en Educación Secundaria. Actualmente está realizando el doctorado y es becaria de 
investigación del grupo Análisis Didáctico y Comportamental del Deporte (ADICODE). Sus líneas de investigación se centran en las diferencias de género en el deporte. E-mail: ingopo04@alumnos.unex.es

Juan José Pulido González es Licenciado en Ciencias de la Actividad Física y el Deporte por la Universidad de Extremadura, con el premio de "mejor expediente académico de la XIII promoción". Becario de investigación en el grupo Análisis Didáctico y Comportamental del Deporte (ADICODE) de la Facultad de Ciencias del Deporte. Sus líneas de investigación se centran en los motivos de práctica de los agentes deportivos: entrenadores y deportistas. Entrenador Nacional de Ciclismo. Máster universitario en Investigación en Ciencias Sociales y Jurídicas Ciencias del Deporte y Máster Universitario en Educación Secundaria. E-mail: jpulidog@alumnos.unex.es

Eduardo M. Cervelló Gimeno es Catedrático de Universidad en el Área de Educación Física y Deportiva, Licenciado en Ciencias de la Actividad Física y el Deporte, Doctor en Psicología, autor de más de cien artículos de investigación en revistas internacionales, y Director del Centro de Investigación del Deporte de la Universidad Miguel Hernández de Elche. E-mail: ecervello@umh.es 